\title{
Artigo Original / Original Paper \\ Estoque e perda de necromassa da vegetação lenhosa em um gradiente fitofisionômico na transição Amazônia-Cerrado
}

\author{
Stock and loss of necromass of trees on a vegetation gradient in the Amazon-Cerrado transition
}

\author{
Karla Monique Silva Carneiro ${ }^{1}$, Simone Matias Reis ${ }^{2,5}$, Paulo Sérgio Morandi ${ }^{2}$, Fernando Elias ${ }^{3,4}$, \\ Edmar Almeida de Oliveira ${ }^{2}$, Ben Hur Marimon-Junior ${ }^{1}$ \& Beatriz Schwantes Marimon ${ }^{1}$
}

\begin{abstract}
Resumo
A necromassa é um importante componente da decomposição e ciclagem de nutrientes nos ecossistemas naturais. Nosso objetivo foi avaliar o estoque e perda de necromassa da comunidade e das espécies em um gradiente fitofisionômico de cerrado típico, cerradão e floresta estacional semidecidual, na transição Amazônia-Cerrado. Calculamos, para cada fitofisionomia, o estoque de necromassa, por meio dos diâmetros dos indivíduos mortos em pé em 2005, 2008, 2011 e 2013. Também calculamos a perda de necromassa da comunidade e das espécies por meio dos diâmetros dos indivíduos que permaneceram mortos em pé entre, pelo menos, dois censos (2002-2005; 2005-2008; 2008-2011 ou 2011-2013). O cerrado típico apresentou estoque e perda de necromassa significativamente inferior ao cerradão e à floresta estacional. Ao nível de espécie, a taxa de decomposição de necromassa não apresentou correlação com a densidade da madeira. Maiores estoque e perda de necromassa na floresta estacional em relação ao cerrado típico podem estar relacionados ao maior porte dos indivíduos, que pode propiciar maior cobertura do dossel e microclima mais favorável à ação de organismos decompositores. Dessa forma, características intrínsecas de cada fitofísionomia podem estar relacionadas diretamente ao estoque e perda de necromassa em formações savânicas e florestais da transição Amazônia-Cerrado.

Palavras-chave: ciclagem de nutrientes, decomposição, densidade da madeira, biomassa, estoque de carbono.
\end{abstract}

\begin{abstract}
Necromass is an important component of the decomposition and nutrients cycling in natural ecosystems. Our objective was to evaluate the stock and loss of necromass of the community and of the species in a vegetation gradient of cerrado típico, cerradão and floresta estacional semidecidual, in the Amazônia-Cerrado transition. We estimate, for each vegetation type, the necromass stock, through the diameters of the dead standing trees in 2005, 2008, 2011 and 2013. We also estimate the loss of necromass at both, community and species level, through the diameters of trees who remained standing dead between at least two inventories (2002-2005, 2005-2008, 2008-2011 or 2011-2013). The cerrado típico showed lower stock and loss of necromass than cerradão and floresta estacional. At the species level, there was no relationship between necromass decomposition rates and wood density. The greater stock and loss of necromass in the floresta estacional semidecidual in relation to the cerrado típico can be related to the greater size of the trees, which can provide more canopy cover and favorable microclimate to the action of decomposing organisms. Thus, intrinsic characteristics of each vegetation type may be directly related to the stock and loss of necromass in savanna and forest formations of the Amazônia-Cerrado transition.
\end{abstract}

Key words: nutrient cycling, decomposition, wood density, biomass, carbon stock.

\footnotetext{
${ }^{1}$ Universidade do Estado de Mato Grosso, Prog. Pós-graduação em Ecologia e Conservação, Lab. Ecologia Vegetal, BR-158, km 655, C.P. 08, 78690-000, Nova Xavantina, MT, Brasil.

${ }^{2}$ Universidade do Estado de Mato Grosso/Universidade Federal do Amazonas, Prog. Pós-graduação da Rede de Biodiversidade e Biotecnologia da Amazônia Legal, Rede BIONORTE, BR-158, km 655, C.P. 08, 78690-000, Nova Xavantina, MT, Brasil.

${ }^{3}$ Universidade Federal do Pará/Embrapa Amazônia Oriental, Prog. Pós-graduação em Ecologia, Inst. Ciências Biológicas, R. Augusto Corrêa 01, 66095-930, Campus Guamá, Belém, PA, Brasil.

${ }^{4}$ ORCID: <https://orcid.org/0000-0001-9190-1733>

${ }^{5}$ Autor para correspondência: simonematiasreis@gmail.com
} 


\section{Introdução}

As florestas tropicais são grandes reservatórios de carbono, podendo estocar cerca de $40 \%$ de todo o carbono terrestre (Malhi \& Grace 2000). O Cerrado, assim como as florestas, apresenta elevada capacidade de sequestro de carbono (Corazza et al. 1999; Lopes \& Miola 2010). Entretanto, essa capacidade vem sendo reduzida, especialmente na extensa zona de transição Amazônia-Cerrado, coincidente com o "arco do desmatamento", onde o avanço da agropecuária tem transformado a vegetação natural em áreas de pastagens e cultivos agrícolas (Fearnside 2005; Nogueira et al. 2008; Alencar et al. 2015). Em áreas preservadas, o carbono retido nas árvores é liberado lentamente pela decomposição da necromassa (Brow 1997), contribuindo para a manutenção da produtividade dos ecossistemas (Barbosa et al. 2009). A necromassa é, por definição, toda biomassa de madeira morta em pé ou caída presente em troncos de árvores, galhos e folhas provenientes de elementos lenhosos (Harmon et al. 1986; Keller et al. 2004). Esse compartimento funciona como habitat de muitos organismos, como bactérias, fungos e invertebrados (Nordén \& Paltto 2001). Além disso, a necromassa reduz a erosão do solo (Enrong et al. 2006), auxilia no armazenamento de água, energia e nutrientes, favorecendo o desenvolvimento da cobertura vegetal superficial (Harmon et al. 1986; Sayer et al. 2006). A necromassa também é, um importante componente para o estoque de carbono, sendo responsável por $8 \%$ do carbono nas florestas do mundo (Pan et al. 2011). Nas florestas tropicais, ela representa de 20 a 40\% do carbono estocado (Brown 1997; Palace et al. 2007) e está relacionada diretamente com o total de biomassa acima do solo e com a taxa de mortalidade lenhosa (Chao et al. 2009). Apesar da elevada importância da necromassa, até o presente momento não há estudos que avaliaram o estoque e a perda de necromassa em diferentes fitofisionomias da transição Amazônia-Cerrado no sul da Amazônia.

$\mathrm{Na}$ transição Amazônia-Cerrado, são observadas diversas formações vegetacionais, tais como, cerrado típico, cerradão e floresta estacional semidecidual (Ratter et al. 2003; Marimon-Junior \& Haridasan 2005; Marimon et al. 2014). O cerrado típico caracteriza-se pela presença predominante da vegetação arbóreaarbustiva, com cobertura de dossel entre 20 a $50 \%$ e altura entre três e seis metros (Ribeiro
\& Walter 2008). Segundo os mesmos autores, o cerradão apresenta cobertura arbórea entre 50 a $90 \%$ e altura entre 8 a $15 \mathrm{~m}$, apresentando um padrão tipicamente florestal. A floresta estacional semidecidual (Veloso et al. 1991) apresenta árvores variando ente 15 a $25 \mathrm{~m}$ e cobertura de dossel entre 70 a $95 \%$ no período chuvoso. No entanto, no período de seca, a cobertura da vegetação pode ser inferior a $50 \%$ (Veloso et al. 1991; Ribeiro \& Walter 2008). O cerrado típico possui menor biomassa e taxa de mortalidade que o cerradão e ambas fitofisionomias possuem menor biomassa e mortalidade que a floresta estacional semidecidual (Marimon et al. 2014). Dessa forma, espera-se que o cerrado típico também apresente menor estoque de necromassa que o cerradão e a floresta estacional, uma vez que o estoque de necromassa está diretamente relacionado a biomassa e mortalidade (Chao et al. 2009).

Após a morte, os troncos das árvores podem permanecer durante muitos anos sem se decompor ou em processo lento de decomposição (Toledo 2009). O tempo de residência do carbono, contido na biomassa da vegetação, é um dos principais indicadores da velocidade de ciclagem do carbono (Malhi et al. 2004). Portanto, períodos longos de residência podem significar uma dinâmica mais lenta, acompanhada de uma baixa produtividade anual de biomassa (Stadtler 2007). Esse processo pode variar conforme os tipos de vegetação e características estruturais e funcionais das espécies, tal como a densidade da madeira (Luccas 2011). Espécies com menor densidade da madeira podem ser decompostas em um período de tempo mais curto (Chambers et al. 2000; Chao et al. 2009), devido a menor produção de celulose, lignina, hemicelulose e células parenquimatosas em relação às espécies de alta densidade da madeira (De Paula 2005).

Outros fatores, tais como precipitação, temperatura e umidade relativa do ar, podem influenciar na decomposição da necromassa (Palace et al. 2008). A umidade relativa do ar geralmente é menor em áreas cobertas por savanas do que em áreas florestais (Hoffmann et al. 2012), e esse fator pode retardar o tempo de decomposição da necromassa da vegetação savânica (Luccas 2011). No caso da vegetação da transição Amazônia-Cerrado, esta ocorre em uma região que apresenta clima sazonal bem marcado e está sujeita a eventos de seca e prolongamento na estiagem (Lewis et al. 2011; Fu et al. 2013), o que pode favorecer o processo de decomposição. 
Adicionalmente, a vegetação da zona de transição tem sofrido frequentes distúrbios gerados pelo fogo, especialmente no período de seca, o qual pode reduzir o estoque de biomassa da vegetação lenhosa (Balch et al. 2011; Brando et al. 2014).

Diante do exposto, nosso objetivo foi avaliar o estoque e perda de necromassa da vegetação lenhosa em um gradiente fitofísionômico na transição Cerrado-Amazônia. Procuramos responder as seguintes perguntas: 1) o estoque de necromassa varia entre ambientes savânicos (cerrado típico) e florestais (cerradão e floresta estacional semidecidual)? 2) a perda de necromassa varia entre as formações savânicas e florestais? Testamos as hipóteses de que o cerradão e a floresta estacional semidecidual exibem maior: i) estoque de necromassa total e, ii) perda de necromassa do que o cerrado típico, devido ao maior porte das árvores e da biomassa viva estocada nessas florestas, em relação ao cerrado típico (Franczak et al. 2011; Mews et al. 2011a, b; Marimon et al. 2014). Também avaliamos se a perda de necromassa está relacionada com a densidade da madeira (característica intrínseca da espécie). Nesse caso, testamos a hipótese de que iii) as espécies com menor densidade da madeira são decompostas em um período de tempo mais curto em relação às espécies de maior densidade (Chambers et al. 2000; Chao et al. 2009).

\section{Material e Métodos}

Área de estudo

Realizamos esse estudo em um gradiente fitofisionômico na transição Amazônia-Cerrado, compreendendo um cerrado típico (1442'26,2”'S,

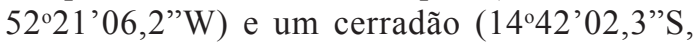
$52^{\circ} 21^{\prime} 02,6$ 'W), localizados no Parque Municipal do Bacaba e uma floresta estacional semidecidual (1449'32"S, 5206'20"W) na Fazenda Vera Cruz, no município de Nova Xavantina, leste do estado de Mato Grosso, Brasil.

O clima da região onde as parcelas estão localizadas é do tipo $A w$ de Köppen (Alvares et al. 2013) com dois períodos bem definidos, um seco (abril a setembro) e outro chuvoso (outubro a março). Os solos predominantes são Latossolo Vermelho-Amarelo e Latossolo Amarelo, distróficos, com textura média, bem drenados, profundos e relevo plano (MarimonJunior \& Haridasan 2005). A precipitação anual é de aproximadamente $1.500 \mathrm{~mm}$, com temperaturas médias de $25^{\circ} \mathrm{C}$ (Marimon et al. 2010).
Coleta de dados

Em 2002, estabelecemos 50 parcelas permanentes de $10 \times 10 \mathrm{~m}$ nas áreas de cerrado típico e de cerradão (Marimon-Junior \& Haridasan 2005) e, em 2003, em uma floresta estacional (Marimon et al. 2014), totalizando 0,5 ha em cada área. Em cada parcela, mensuramos o diâmetro e altura de todos os indivíduos vivos com diâmetro a altura do solo $\left(\mathrm{DAS}_{0,30 \mathrm{~m}}\right) \geq 5 \mathrm{~cm}$, identificamos e marcamos com placas de alumínio. Para a floresta estacional, adotamos como limite de inclusão o diâmetro à altura do peito $\left(\mathrm{DAP}_{1,30 \mathrm{~m}}\right)$. A altura foi medida com trena laser Leica DISTO. Remedimos todos os indivíduos vivos e mortos que permaneceram em pé em 2005, 2008, 2011 e 2013 no cerrado típico e cerradão em 2008, 2011 e 2013 na floresta estacional (Mews et al. 2011b; Franczak et al. 2011; Marimon et al. 2014; Reis et al. 2015). Em 2008, uma queimada acidental (antrópica) atingiu todas as parcelas do cerrado típico e 15 parcelas do cerradão $(30 \%)$. A floresta estacional não foi atingida pelo fogo durante o intervalo de estudo. Os indivíduos mortos caídos não foram remedidos, em função da dificuldade de identificarmos qual árvore era, especialmente nas parcelas atingidas pelo fogo.

Na primeira medição, coletamos amostras de material botânico das espécies para identificação e inclusão na coleção científica do Herbário NX da Universidade do estado de Mato Grosso, Campus de Nova Xavantina. Adotamos o sistema de classificação sugerido por APG IV (2016) para a classificação das famílias. Revisamos e atualizamos a nomenclatura dos táxons através do banco de dados do BFG (2018).

Para o presente estudo, utilizamos apenas os indivíduos que morreram após a primeira medição e que foram identificados na primeira medição.

\section{Análise de dados}

Estimamos o estoque de necromassa (para todos os censos, exceto para o primeiro) com duas equações específicas, a primeira para o cerrado típico e cerradão [e $(-2+2,42 * \operatorname{Ln}(D A S))]$ (Chave et al. 2001) e a segunda para a floresta estacional $\left(0.0673 *\left(D M * D A P^{\wedge} 2 * H\right)^{\wedge} 0.976\right)$ (Chave et al. 2014). Onde: $D A S=$ diâmetro à altura do solo, $D M$ $=$ densidade da madeira, $D A P=$ diâmetro a altura do peito e $H=$ altura. Obtivemos os dados de densidade da madeira em Roberto (2014) e no banco de dados ForestPlots.net, o qual apresenta informações para espécies neotropicais (<https://www.ForestPlots. net/>; Chave et al. 2009). 
Com base no estoque de necromassa, calculamos a perda de necromassa, para a comunidade bem como para as espécies, entre os períodos de 2005-2008, 2008-2011 e 2011-2013 no cerrado típico e cerradão e entre 2008-2011 e 2011-2013 para a floresta estacional. Adaptamos a equação de Guimarães et al. (2008), utilizada para calcular a perda de área basal, para calculamos a perda de necromassa $(P=\{1-[(N O-N f) / \mathrm{N} 0] 1 / t\} \times$ $100)$, onde $P=$ perda, $N O$ e $N f$ são os estoques de necromassa inicial e final, respectivamente, e $t$ é o tempo transcorrido entre os anos. Calculamos a perda de necromassa apenas para os indivíduos que permaneceram mortos em pé entre pelo menos dois censos. Para as espécies, calculamos a perda de necromassa apenas para os indivíduos que foram identificados no primeiro censo, ou seja, estavam vivos no primeiro inventário e depois morreram. Não foi possível incluir nas análises a perda de necromassa, entre 2008 e 2011, para o cerrado típico, uma vez que apenas três indivíduos permaneceram em pé após a queimada.

Para verificar se o estoque de necromassa se diferia entre as fitofisionomias, em cada ano (ex. CT $2008 \times$ CD $2008 \times$ FE 2008), utilizamos uma análise de variância (ANOVA) com post hoc de Tukey (Zar 2010). Para compararmos a perda de necromassa entre as fitofisionomias em cada intervalo de tempo (ex. CT 2008-2013 $\times$ CD 2008-2013 × FE 2008-2013), utilizamos a análise de Kruskal-Wallis com post hoc de Dunnet (Zar 2010). Dentro de cada fitofisionomia, utilizamos uma análise de correlação de Spearman para verificarmos se a taxa de decomposição das espécies estava correlacionada com a densidade da madeira (Zar 2010). Adotamos o nível de 5\% de significância para todas as análises realizadas.

\section{Resultados}

\section{Estoque de necromassa}

O cerrado típico apresentou estoque de necromassa inferior ao cerradão e a floresta estacional em todos os intervalos avaliados (Fig. 1). Por outro lado, o cerradão e a floresta estacional foram similares em todos os períodos avaliados (Fig. 1). No cerrado típico, encontramos $42(6,8 \%), 36(5,8 \%), 17(3,7 \%)$ e $50(4,5 \%)$ indivíduos mortos em 2005, 2008, 2011 e 2013, respectivamente. Para esses mesmos anos, observamos no cerradão $84(8,6 \%), 87(9,5 \%), 111$ $(16,1 \%)$ e $63(9,6 \%)$ indivíduos mortos. Na floresta estacional, encontramos $76(16,7 \%), 63(14,5 \%)$ e $63(15 \%)$ em 2008, 2011 e 2013, respectivamente.

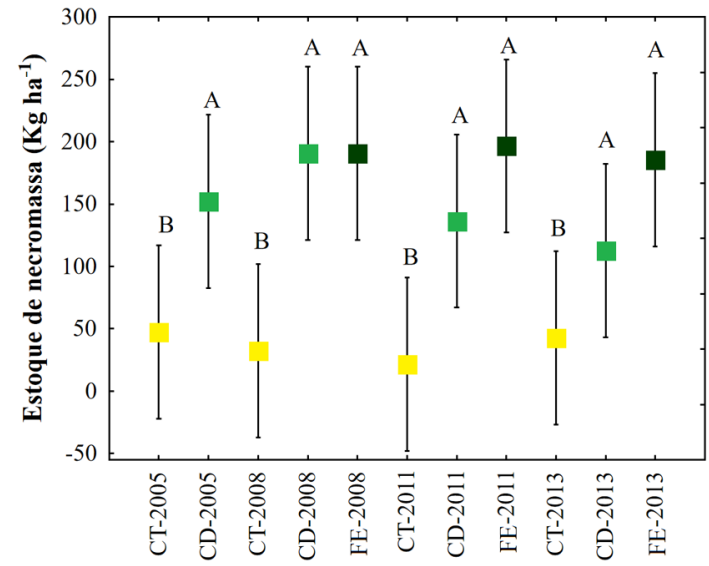

Figura 1 - Estoque de necromassa $\left(\mathrm{kg} \mathrm{ha}^{-1}\right)$ para o cerrado típico (CT; quadrado amarelo), cerradão (CD; quadrado verde claro) e floresta estacional semidecidual (FE; quadrado verde escuro) entre 2005 e 2013 em Nova Xavantina, Mato Grosso. Médias seguidas de mesma letra significa que as fitofisionomias não se diferem significativamente entre si em cada ano (ex. CT-08 $\times$ CD-08 $\times$ FE-08); $F_{(10,539)}=3,996, p<0,0001$; as barras verticais indicam intervalo de confiança de 0,95 .

Figure 1 - Necromass stock $\left(\mathrm{kg} \mathrm{ha}^{-1}\right)$ for the cerrado típico (CT; yellow square), cerradão (CD; light green square) and floresta estacional semidecidual (FE; dark green square) between 2005 and 2013 in Nova Xavantina, Mato Grosso. Means followed by the same letter means that the vegetation types do not differ significantly from each other in each year (e.g. CT- $08 \times$ CD-08 $\times$ FE-08); $F_{(10,539)}=3,996, p<0,0001$; vertical bars denote 0.95 confidence intervals.

\section{Perda de necromassa}

ao nível de comunidade

O cerrado típico apresentou perda de necromassa inferior ao cerradão e a floresta estacional entre 2011 e 2013, porém foi similar ao cerradão entre 2005 e 2008 (Fig. 2). O cerradão e a floresta estacional apresentaram perda de necromassa similares em todos os períodos avaliados (Fig. 2). No cerrado típico, no intervalo em que houve a passagem do fogo, entre 2008 e 2011, apenas três indivíduos permaneceram mortos em pé, os demais caíram ou foram consumidos pelo fogo.

\section{Perda de necromassa}

ao nível de espécie

Ao nível de espécie, a perda de necromassa não apresentou correlação significativa com a densidade da madeira em nenhuma das fitofisionomias (Correlação de Spearman: CT = 


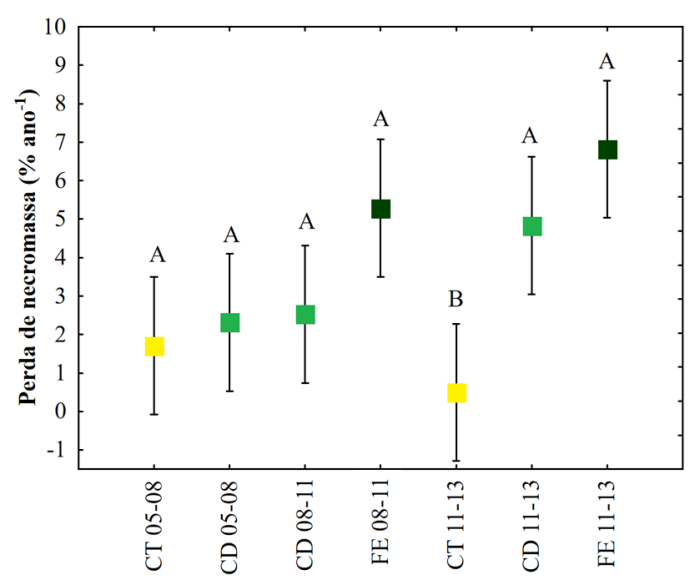

Figura 2 - Perda de necromassa $\left(\%\right.$ ano $\left.^{-1}\right)$ em cerrado típico (CT; quadrado amarelo), cerradão (CD; quadrado verde claro) e floresta estacional semidecidual (FE; quadrado verde escuro) entre os anos de 2005 e 2008 (05-08), 2008 e 2011 (08-11), e 2011 e 2013 (11-13) em Nova Xavantina, Mato Grosso. Médias seguidas de mesma letra indicam que a taxa de perda não se difere significativamente entre as fitofisionomias em cada intervalo de tempo (ex. CT 11-13 × CD 11-13 × FE $11-13) ; \mathrm{n}=$ número de indivíduos.

Figure 2 - Necromass loss $\left(\%\right.$ year $\left.{ }^{-1}\right)$ for the cerrado típico (CT; yellow square), cerradão (CD; light green square) and floresta estacional semidecidual (FE; dark green square) between 2005 and 2008 (05-08), 2008 and 2011 (08-11), and 2011 and 2013 (11-13) in Nova Xavantina, Mato Grosso. Medians followed by the same letter indicates that the loss rate did not differ significantly between the vegetation types at each time interval (e.g. CT 11-13 $\times$ CD 11-13 × FE 11-13); $\mathrm{n}=$ number of individuals.

$-0,10 ; \mathrm{CD}=0,02$ e $\mathrm{FE}=-0,18 ; p>0,05)$. Apesar da ausência de correlação com a densidade da madeira, a taxa de perda de necromassa variou entre os intervalos, entre as espécies e, inclusive, dentro dos indivíduos de uma mesma espécie (Tab. 1). No cerrado típico, as espécies que apresentaram maior perda de necromassa foram Dimorphandra mollis Benth. e Qualea parviflora Mart. (2005-2008), seguidas por Mimosa laticifera Rizzini \& A. Mattos $(2008,2011)$ (Tab. 1). As espécies Annona coriacea Mart., Hymenaea stigonocarpa Mart. ex Hayne, Lafoensia pacari A. St.-Hil. e Machaerium acutifolium Vogel não apresentaram perda de necromassa e, Q. grandiflora Mart. apresentou a menor perda (Tab. 1). No cerradão, Tapirira guianensis Aubl., Mezilaurus crassiramea (Meisn.) Taub. ex Mez, Cordiera sessilis (Vell.) Kuntze e Tachigali vulgaris L.G. Silva \& H.C. Lima se destacaram entre as espécies com maior perda de necromassa (Tab. 1). Por outro lado, Hirtella glandulosa Spreng. se destacou entre as espécies que menos perderam necromassa. Na floresta estacional, Diospyros sericea A. DC. e Cupania vernalis Cambess. se destacaram entre as espécies que mais perderam necromassa e Mouriri apiranga Spruce ex Triana apresentou a menor perda (Tab. 1).

\section{Discussão}

Nossos resultados mostram que o estoque e a perda da necromassa variam entre as fitofisionomias e entre diferentes espécies. As formações florestais exibem maiores estoques de necromassa do que o cerrado típico, confirmando nossa primeira hipótese. O cerrado típico apresentou perda de necromassa significativamente inferior à floresta estacional e ao cerradão entre 2011 e 2013, entretanto foi similar ao cerradão entre 2005 e 2008, refutando parcialmente nossa segunda hipótese. Além disso, ao nível de espécie, não observamos correlação entre taxa de perda de necromassa e densidade da madeira em nenhuma das três fitofisionomias, refutando nossa terceira hipótese.

\section{Estoque de necromassa}

O maior estoque de necromassa nas comunidades florestais pode estar relacionado com o maior aporte de biomassa viva e com a maior taxa de mortalidade nessas áreas em relação ao cerrado típico (Marimon et al. 2014). Em todos os períodos avaliados, observamos maior percentual de mortos no cerradão e na floresta estacional, o que favorece maior entrada de necromassa nessas fitofisionomias, corroborando com os achados de Chao et al. (2009), em um gradiente ambiental na Amazônia. Adicionalmente, a sucessão da composição de espécies ao longo do tempo também conduz ao aumento na necromassa acumulada, devido ao adensamento da vegetação e a substituição de espécies de pequeno porte por espécies de maior porte e que, geralmente, possuem maiores diâmetros (Mota \& Torezan 2013), complementando os achados deste estudo.

Apenas três indivíduos permaneceram mortos em pé após a passagem do fogo no cerrado típico, sugerindo que o fogo pode potencializar a decomposição da necromassa e reduzir o estoque de necromassa. Dos três indivíduos que permaneceram em pé, dois eram de Mimosa laticifera, a qual apresenta elevada densidade da madeira $\left(0,80 \mathrm{~g} \mathrm{~cm}^{-3}\right)$, e foram menos afetados pela queimada do que indivíduos de espécies de menor densidade da madeira. Dessa forma, queimadas, 
Tabela 1 - Taxa anual média de perda de necromassa (TP) $\left(\%\right.$ ano $\left.{ }^{-1}\right)$ para os indivíduos que permaneceram em pé entre 2005 e 2008 (05-08), 2008 e 2011 (08-11) e 2011 e 2013 (11-13) das espécies de cerrado típico (CT), cerradão (CD) e floresta estacional semidecidual (FE), Nova Xavantina, Mato Grosso. As espécies foram listadas em ordem crescente do gradiente fitofisionômico CT, $\mathrm{CD}$ e FE. Fito $=$ Fitofisionomia, $\mathrm{DM}=$ densidade da madeira, entre parênteses () = número de indivíduos.

Table 1 - Average annual necromass loss rate (TP) $\left(\%\right.$ ano $\left.^{-1}\right)$ for individuals who remained standing between 2005 and 2008 (05-08), 2008 and 2011 (08-11) and 2011 and 2013 (11-13) of the species of the cerrado típico (CT), cerradão (CD) and floresta estacional semidecidual (FE), Nova Xavantina, Mato Grosso. The species were listed in ascending order of the phytophysiognomic gradient (CT), $(\mathrm{CD})$ and $(\mathrm{FE})$. Fito $=$ vegetation type, $\mathrm{WD}=$ wood density, between parenthesis ()$=$ number of individuals.

\begin{tabular}{|c|c|c|c|c|c|}
\hline Fito & Espécies & $\mathrm{DM}\left(\mathrm{g} \mathrm{cm}^{-3}\right)$ & TP 05/08 (\%) & TP 08/11 (\%) & TP 11/13 (\%) \\
\hline $\mathrm{CT}$ & Annona coriacea Mart. & 0.58 & - & - & $0.0(1)$ \\
\hline $\mathrm{CT}$ & Byrsonima pachyphylla A. Juss. & 0.66 & $4.3(2)$ & - & $7.5(1)$ \\
\hline CT & Couepia grandiflora (Mart. \& Zucc.) Benth. & 0.56 & $2.5(2)$ & - & - \\
\hline $\mathrm{CT}$ & Dimorphandra mollis Benth. & 0.61 & $22.6(1)$ & - & - \\
\hline $\mathrm{CT}$ & Erythroxylum suberosum A. St.-Hil. & 0.63 & - & - & $6.1(1)$ \\
\hline $\mathrm{CT}$ & Eugenia aurata $\mathrm{O}$. Berg & 0.64 & $9.1(2)$ & - & $0.0(1)$ \\
\hline $\mathrm{CT}$ & Hymenaea stigonocarpa Mart. ex Hayne & 0.77 & $0.0(1)$ & - & - \\
\hline $\mathrm{CT}$ & Lafoensia pacari A. St.-Hil. & 0.70 & - & - & $0.0(1)$ \\
\hline $\mathrm{CT}$ & Machaerium acutifolium Vogel & 0.77 & - & - & $0.0(1)$ \\
\hline $\mathrm{CT}$ & Mimosa laticifera Rizzini \& A. Mattos & 0.80 & $0.3(1)$ & $15.9(2)$ & $3.6(4)$ \\
\hline $\mathrm{CT}$ & Qualea grandiflora Mart. & 0.70 & $0.6(1)$ & - & $0.0(1)$ \\
\hline $\mathrm{CT}$ & Qualea multiflora Mart. & 0.65 & $4.9(1)$ & - & - \\
\hline $\mathrm{CT}$ & Qualea parviflora & 0.66 & $17.8(1)$ & - & $9.8(1)$ \\
\hline $\mathrm{CD}$ & Aspidosperma multiflorum A. DC. & 0.69 & $0.0(1)$ & - & $15.0(2)$ \\
\hline $\mathrm{CD}$ & Cordiera sessilis (Vell.) Kuntze & 0.88 & $20.0(1)$ & - & $14.6(1)$ \\
\hline $\mathrm{CD}$ & Dimorphandra mollis Benth. & 0.61 & $6.5(2)$ & $7.9(2)$ & $2.6(1)$ \\
\hline $\mathrm{CD}$ & Emmotum nitens (Benth.) Miers & 0.76 & $3.3(1)$ & 6.1(1) & - \\
\hline $\mathrm{CD}$ & Euplassa inaequalis (Pohl) Engl. & 0.62 & - & $5.8(1)$ & - \\
\hline $\mathrm{CD}$ & Guapira graciliflora (Mart. ex Schmidt) Lundell & 0.46 & $0.3(1)$ & 7.3(1) & - \\
\hline $\mathrm{CD}$ & Heisteria ovata Benth. & 0.67 & - & $4.0(1)$ & - \\
\hline $\mathrm{CD}$ & Hirtella glandulosa Spreng. & 0.68 & $0.3(1)$ & - & $0.0(1)$ \\
\hline $\mathrm{CD}$ & Luetzelburgia praecox (Harms) Harms & 0.76 & - & $2.9(1)$ & $12.0(1)$ \\
\hline $\mathrm{CD}$ & Matayba guianensis Aubl. & 0.72 & $9.9(1)$ & - & - \\
\hline $\mathrm{CD}$ & Mezilaurus crassiramea (Meisn.) Taub. ex Mez & 0.68 & - & $0.4(1)$ & $29.7(3)$ \\
\hline $\mathrm{CD}$ & Myrcias plendens (Sw.) DC. & 0.66 & 3.1(3) & - & - \\
\hline $\mathrm{CD}$ & Niedenzuella glabra (Spreng.) W.R. Anderson & 0.00 & $10.4(1)$ & - & - \\
\hline $\mathrm{CD}$ & Qualea grandiflora Mart. & 0.70 & - & $2.9(1)$ & - \\
\hline $\mathrm{CD}$ & Qualea multiflora Mart. & 0.65 & $15.8(2)$ & - & - \\
\hline $\mathrm{CD}$ & Roupala montana Aubl. & 0.73 & - & $2.3(4)$ & $14.1(5)$ \\
\hline $\mathrm{CD}$ & Tachigali vulgaris L.G. Silva \& H.C. Lima & 0.61 & $7.6(5)$ & $12.0(6)$ & $14.2(6)$ \\
\hline $\mathrm{CD}$ & Vatairea macrocarpa (Benth.) Ducke & 0.68 & - & $0.4(1)$ & - \\
\hline
\end{tabular}




\begin{tabular}{|c|c|c|c|c|c|}
\hline Fito & Espécies & DM $\left(\mathrm{g} \mathrm{cm}^{-3}\right)$ & TP 05/08 (\%) & TP 08/11 (\%) & TP 11/13 (\%) \\
\hline $\mathrm{CD}$ & Xylopia aromatica (Lam.) Mart. & 0.59 & - & $0.1(1)$ & - \\
\hline $\mathrm{CD}$ & Tapirira guianensis Aubl. & 0.67 & - & - & $30.2(1)$ \\
\hline $\mathrm{FE}$ & Amaioua guianensis Aubl. & 0.67 & - & 5.1(7) & $3.3(2)$ \\
\hline $\mathrm{FE}$ & Buchenavia tetraphylla (Aubl.) R.A. Howard & 0.62 & - & 7.8(1) & $15.3(1)$ \\
\hline $\mathrm{FE}$ & Caraipa indet & 0.60 & - & $3.5(2)$ & - \\
\hline FE & Chaetocarpus echinocarpus (Baill.) Ducke & 0.79 & - & $4.5(1)$ & $0(1)$ \\
\hline FE & Cheiloclinium cognatum (Miers) A.C. Sm. & 0.63 & - & $10.8(6)$ & $10.3(6)$ \\
\hline FE & Cupania vernalis Cambess. & 0.65 & - & $9.1(1)$ & $46.4(1)$ \\
\hline $\mathrm{FE}$ & Diospyros sericea A. DC. & 0.69 & - & $22.0(1)$ & $40.8(1)$ \\
\hline $\mathrm{FE}$ & Hirtella hispidula Miq. & 0.78 & - & - & $7.8(1)$ \\
\hline FE & Hirtella gracilipes (Hook.f.) Prance & 0.79 & - & $7.0(2)$ & - \\
\hline $\mathrm{FE}$ & Inga heterophylla Willd. & 0.73 & - & $10.7(2)$ & $22.1(2)$ \\
\hline FE & Mabea fistulifera Mart. & 0.65 & - & 7.4(3) & 19.1(2) \\
\hline FE & Minquartia guianensis Aubl. & 0.83 & - & $3.8(3)$ & $7.8(5)$ \\
\hline FE & Mollia lepidota Spruce ex Benth. & 0.49 & - & - & $1.1(1)$ \\
\hline FE & Mouriri apiranga Spruce ex Triana & 0.83 & - & $0(1)$ & $0.2(1)$ \\
\hline FE & Nectandra cuspidata Nees & 0.57 & - & $10.3(2)$ & - \\
\hline FE & Nectandra hihua (Ruiz \& Pav.) Rohwer & 0.42 & - & $8.6(14)$ & $14.3(7)$ \\
\hline FE & Sacoglottis guianensis Benth. & 0.77 & - & - & $3.1(1)$ \\
\hline FE & Terminalia indet & 0.55 & - & $4.7(1)$ & $7.5(1)$ \\
\hline FE & Tetragastris altissima (Aubl.) Swart & 0.71 & - & 3.4(3) & $2.3(4)$ \\
\hline
\end{tabular}

além de afetar a estrutura, a composição florística e a dinâmica da comunidade (Felfili 1998; Hoffmann et al. 2012; Gomes et al. 2014), interferem no processo de ciclagem de nutrientes (Redin et al. 2011), e podem atuar de maneira mais direta sobre as espécies que apresentam menor densidade da madeira, reduzindo o estoque de necromassa. Assim, as queimadas podem acelerar o processo de perda de necromassa, reduzindo diretamente o estoque de necromassa, atuando, principalmente, nas espécies que apresentam menor densidade da madeira.

\section{Perda de necromassa}

ao nível de comunidade

Maiores perdas de necromassa nas fitofisionomias florestais, podem estar relacionadas com as características do hábitat, as quais podem potencializar a decomposição. Por exemplo, as florestas possuem dossel fechado, refletindo em menor entrada de luz, redução da temperatura e aumento da umidade, possibilitando um microclima mais favorável ao processo de decomposição (Weedon et al. 2009; Rebetez et al. 2012; Hoffmann et al. 2012; Mota \& Torezan 2013). Por outro lado, a umidade relativa do ar, principalmente durante o dia, é mais baixa em ambientes savânicos (Hoffmann et al. 2012), o que pode retardar a perda da necromassa no cerrado típico (Palace et al. 2008; Luccas 2011; Mota \& Torezan 2013).

Em um estudo recente, desenvolvido em áreas de cerrado típico e cerradão, Oliveira et al. (2016) observaram que a ciclagem de nutrientes na serapilheira (folhas, galhos finos, flores) é mais acelerada no cerradão que no cerrado típico. Para os autores, tal achado pode estar relacionado com uma guilda de espécies mais complexa no cerradão, além de um dossel mais contínuo, especialmente em função da ocorrência de lianas, as quais contribuem para uma maior cobertura de dossel e 
microclima favorável à ação de microrganismos decompositores. Tal explicação também ajuda entender o fato de termos encontrado maior perda de necromassa no cerradão do que no cerrado típico.

Adicionalmente, a sazonalidade climática da região e os recorrentes eventos de seca (Lewis et al. 2011), são fatores que podem influenciar na perda de necromassa (Saiter et al. 2009). Durante a estiagem a vegetação perde parte de suas folhas e isso aumenta a espessura da camada de serapilheira no solo. Em seguida, durante o período chuvoso, a maior umidade do solo interfere diretamente na atividade dos microrganismos, acelerando, não apenas a decomposição da camada de serapilheira, mas também a decomposição da necromassa, principalmente nas fitofisionomias florestais, nas quais o aporte de serapilheira é maior em relação às savânicas (Valadão et al. 2016; Oliveira et al. 2016).

\section{Perda de necromassa}

ao nível de espécie

Fatores intrínsecos, como a densidade da madeira, podem controlar o processo de decomposição das espécies na Amazônia central (Chambers et al. 2000; Chao et al. 2009). Entretanto, não encontramos correlação entre a densidade da madeira e a taxa de decomposição de necromassa em nenhuma das fitofisionomias avaliadas. Assim, outros fatores como diâmetro e altura talvez possam afetar o processo de decomposição (Chambers et al. 2000; Medeiros \& Miranda 2005). Toledo (2009) também não encontrou correlação entre taxa de decomposição de necromassa e densidade da madeira e sugeriu que o processo de decomposição, provavelmente, pode estar mais associado a organismos decompositores generalistas do que com especialistas associados às características intrínsecas das espécies. Na Amazônia central, existe uma ampla variação na densidade da madeira (0,30-0,96 $\mathrm{g} \mathrm{cm}^{-3}$ ) (Chambers et al. 2000). Por outro lado, a variação foi menor $\left(0,42-0,88 \mathrm{~g} \mathrm{~cm}^{-3}\right)$ no presente estudo, além de que poucas espécies estão nos extremos com alta ou baixa densidade, fator que pode explicar a ausência de correlação entre densidade da madeira e decomposição.

Em relação às espécies que permaneceram mortas em pé por mais tempo e, consequentemente mostraram menor liberação de necromassa, ao longo do tempo, nas três fitofisionomias, destacamos Qualea grandiflora (cerrado típico), Hirtella glandulosa (cerradão) e Mouriri apiranga (floresta estacional). Qualea grandiflora é uma das cinco espécies de maior importância no cerrado típico estudado (Marimon-Junior \& Haridasan 2005) e apresenta ampla ocorrência em todo o Bioma Cerrado (Ratter et al. 2003). A espécie referida também possui elevada suberização no tronco e madeira pesada, com $0,70 \mathrm{~g} \mathrm{~cm}^{-3}$ (Roberto 2014), tornando o lenho mais duro e resistente ao fogo (Do Vale et al. 2002). Hirtella glandulosa, além de apresentar ampla ocorrência em cerradões no leste de Mato Grosso (Marimon-Junior \& Haridasan 2005), é uma espécie de madeira pesada $\left(0,93 \mathrm{~g} \mathrm{~cm}^{-3}\right)$ e apresenta resistência ao fogo (Elias et al. 2013b), fatores que podem contribuir com a sua permanência por maior tempo na comunidade, mesmo depois de morta. Mouriri apiranga é uma espécie arbórea com baixa mortalidade e indicada como uma possível espécie em potencial para uso de áreas degradadas (Mews et al. 2011a). Além disso, $M$. apiranga apresenta alta densidade da madeira $\left(0,83 \mathrm{~g} \mathrm{~cm}^{-3}\right)$, fator que, provavelmente, deve retardar a perda de necromassa. Por outro lado, as espécies Tachigali vulgaris (no cerradão; 0,61 $\left.\mathrm{g} \mathrm{g} \mathrm{cm}^{-3}\right)$ e Tapirira guianensis $\left(0,67 \mathrm{~g} \mathrm{~cm}^{-3}\right)$ estão entre as espécies que mais perderam necromassa. A primeira, apresenta rápido crescimento, ciclo de vida curto (Medeiros 2002; Filizola 2013) e alta sensibilidade a distúrbios (Elias et al. 2013a; Reis et al. 2015), o que pode facilitar sua decomposição. Tapirira guianensis é uma espécie que ocorre em todo território brasileiro, sua madeira é leve e possui baixa resistência a organismos xilófagos tais como cupins (Lorenzi 2002), o que deve favorecer a perda de necromassa.

A necromassa proporciona uma fonte constante de material orgânico para a ciclagem de carbono e nutrientes e contribui para o funcionamento dos ecossistemas (Palace et al. 2012). Nesse contexto, espécies como Tachigali vulgaris e Tapirira guianensis podem ser muito úteis, se o objetivo na recuperação de área degradada for também recuperar o solo, pois estas espécies têm rápida decomposição e podem contribuir no acúmulo de matéria orgânica no solo. Por outro lado, as espécies que apresentaram a menor perda de necromassa ao longo do tempo, como Qualea grandiflora no cerrado típico, Hirtella glandulosa no cerradão e Mouriri apiranga na floresta estacional, apresentam potencial para uso em ações de manejo e recuperação de áreas degradadas, pois podem contribuir diretamente com o processo de fixação do carbono atmosférico, quando vivas, e imobilização, depois de mortas. 
Com base em nossos achados concluímos que o estoque e a perda de necromassa variam em um gradiente fitofisionômico cerrado-cerradãofloresta, sendo que as características intrínsecas de cada fitofisionomia, como cobertura do dossel e umidade do solo, podem estar relacionadas com os parâmetros avaliados. Em geral, as formações florestais apresentam maior estoque e decomposição mais acelerada de necromassa em relação ao cerrado típico. Além disso, distúrbios como o fogo podem alterar o estoque de necromassa e pode variar entre as espécies em função da densidade da madeira e o porte dos indivíduos.

\section{Agradecimentos}

Agradecemos à Fundação de Amparo à Pesquisa do Estado de Mato Grosso (FAPEMAT), a concessão de bolsa de Iniciação Científica à primeira autora, Processo n ${ }^{\circ}$ 586918/2012. Ao Conselho Nacional de Desenvolvimento Científico e Tecnológico (CNPq) CNPq/PELD - Proc. 403725/2012-7 e à FAPEMAT - Proc. 164131/2013, o apoio financeiro ao projeto "Transição Cerrado-Floresta Amazônica: bases ecológicas e socioambientais para a conservação (etapa II)". À Coordenação de Aperfeiçoamento Pessoal de Nível Superior (CAPES), a concessão de bolsa de Estudos a S.M. Reis, P.S. Morandi, E.A. Oliveira e F. Elias; e ao CNPq, a concessão de bolsa de Produtividade em Pesquisa para B.S. Marimon e B.H. Marimon-Junior. À equipe de pesquisa do Laboratório de Ecologia Vegetal (LABEV) da UNEMAT de Nova Xavantina, a ajuda em campo; e aos que de alguma forma contribuíram com este trabalho.

\section{Referências}

Alencar AA, Brando PM, Asner GP \& Putz FE (2015) Landscape fragmentation, severe drought, and the new Amazon forest fire regime. Ecological applications 25: 1493-1505.

Alvares CA, Stape JL, Sentelhas PC, Gonçalves JLM \& Sparovek G (2013) Köppen's climate classification map for Brazil. Meteorologische Zeitschrift, Stuttgart 22: 711-728.

APG IV - Angiosperm Phylogeny Group (2016) An update of the Angiosperm Phylogeny Group classification for the orders and families of flowering plants: APG IV. Botanical Journal of the Linnean Society 181: 1-20.

Balch JK, Nepstad DC, Curran LM, Brando PM, Portela O, Guilherme P, Reuning-Scherer JD \& Carvalho Jr.O (2011) Size, species, and fire behavior predict tree and liana mortality from experimental burns in the Brazilian Amazon. Forest Ecology and Management 261: 68-77.
Barbosa RI, Silva LFSG \& Cavalcante CO (2009) Protocolo necromassa: estoque e produção de Liteira Grossa. Programa de Pesquisa em Biodiversidade, Boa Vista. $23 \mathrm{p}$.

BFG - The Brazil Flora Group (2018) Brazilian Flora 2020: innovation and collaboration to meet Target 1 of the Global Strategy for Plant Conservation (GSPC). Rodriguésia 69: 1513-1527.

Brando PM, Balch JK, Nepstad DC, Morton DC, Putz FE, Coe MT, Silvério D, Macedo MN, Davidson EA, Nóbrega CC, Alencar A \& Britaldo SSF (2014) Abrupt increases in Amazonian tree mortality due to drought-fire interactions. Proceedings of the National Academy of Sciences 111: 6347-6352.

Brown S (1997) Estimating biomass and biomass change of tropical forests: a primer. FAO, Roma. 55p.

Chambers JQ, Higuchi N, Schimel J, Ferreira LV \& Melack JM (2000) Decomposition and carbon cycling of dead trees in tropical forests of central Amazon. Oecologia 122: $380-388$.

Chao KJ, Phillips OL, Baker TR, Peacock J, LopezGonzalez G, Vásquez Martínez R, Monteagudo A \& Torres-Lezama A (2009) After trees die: quantities and determinants of necromass across Amazonia. Biogeosciences 6: 1615-1626.

Chave J, Riéra B \& Dubois M (2001) Estimation of biomass in a neotropical forest of French Guiana: spatial and temporal variability. Journal of Tropical Ecology 17: 79-96.

Chave J, Coomes D, Jansen S, Lewis SL, Swenson NG \& Zanne AE (2009) Towards a worldwide wood economics spectrum. Ecology Letters 12: 351-366.

Chave J, Réjou-Méchain M, Búrquez A, Chidumayo E, Colgan MS, Delitti WBC, Duque A, Eid T, Fearnside PM, Goodman RC, Henry M, Martínez-Yrízar A, Mugasha WA, Muller-Landau HC, Mencuccini M, Nelson BW, Ngomanda A, Nogueira EM, Ortiz-Malavassi E, Pélissier R, Ploton P, Ryan CM, Saldarriaga JG \& Vieilledent G (2014) Improved allometric models to estimate the aboveground biomass of tropical trees. Global Change Biology 20: 3177-3190.

Corazza EJ, Silva JE, Resck DVS \& Gomes AC (1999) Comportamento de diferentes sistemas de manejo como fonte ou depósito de carbono em relação à vegetação do cerrado. Revista Brasileira Ciência do Solo 23: 425-432.

De Paula JE (2005) Caracterização anatômica da madeira de espécies nativas do cerrado, visando sua utilização na produção de energia. Cerne 11: 90-100.

Elias F, Marimon BS, Gomes L, Forsthofer M, Abreu MF, Reis SA, Lenza E, Franczak DD \& Marimon-Junior BH (2013a) Resiliência de um cerradão submetido a perturbações intermediárias na transição CerradoAmazônia. Revista Biotemas 26: 49-62.

Elias F, Marimon BS, Reis SMA, Forsthofer M, Gomes L, Morandi PS \& Marimon-Junior BH(2013b) Dinâmica da distribuição espacial de populações arbóreas, 
ao longo de uma década, em cerradão na transição Cerrado-Amazônia, Mato Grosso. Biota Amazônia 3: $1-14$

Enrong Y, Xihua W \& Jian jun H (2006) Concept and classification of Coarse Wood Debris in Forest Ecosystems. Frontiers in Biology 1: 76-84.

Fearnside PM(2005) Desmatamento na Amazônia brasileira: história, índices e conseqüências. Megadiversidade 1: 113-123.

Felfili JM (1998) Determinação de padrões de distribuição de espécies em uma mata de galeria no Brasil Central com a utilização de técnicas de análise multivariada. Boletim do Herbário Ezechias Paulo Heringer 2: 35-48.

Filizola BDC (2013) Boas práticas de manejo para o extrativismo sustentável da Fava d'Anta. Instituto Sociedade, População e Natureza, Brasília. 76p.

Franczak DD, Marimon BS, Marimon-Junior BH, Mews HA, Maracahipes L \& Oliveira EA (2011) Changes in the structure of a savanna forest over a six-year period in the Amazon-Cerrado transition, Mato Grosso state, Brazil. Rodriguésia 62: 425-436.

Fu R, Yina L, Lib W, Arias PA, Dickinsona RE, Huanga L, Chakrabortya S, Fernandes K, Liebmanne B, Fisherf R \& Mynenig RB (2013) Increased dry-season length over southern Amazonia in recent decades and its implication for future climate projection. Proceedings of the National Academy of Sciences 110: 1811018115.

Gomes L, Maracahipes L, Marimon BS, Reis SM, Elias F, Maracahipes-santos L, Marimon-Junior BH \& Lenza E (2014) Post-fire recovery os savana vegetation from rocky outcrops. Flora 209: 201-2018.

Guimarães JCC, Van den berg E, Castro GC, Machado ELM \& Oliveira-filho AT (2008) Dinâmica do componente arbustivo-arbóreo de uma floresta de galeria aluvial no planalto de Poços de Caldas, MG, Brasil. Revista Brasileira de Botanica 31: 621-632.

Harmon ME, Franklin JF, Swanson FJ, Sollins P, Gregory SV, Lattin JD, Anderson NH, Cline SP, Aumen NG, Sedell JR, Lienkaemper GW, Cromack K \& Cummins KW (1986) Ecology of coarse woody debris in temperate ecosystems. Advances in Ecological Research 15: 133-302.

Hoffmann WA, Jaconis SY, Mckinley KL, Geiger EL, Gotsch SG \& Franco AC (2012) Fuels or microclimate? Understanding the drivers of fire feedbacks at savannaforest boundaries. Austral Ecology 37: 634-643.

Keller M, Palace M, Asner GP, Pereira Junior R \& Silva JNM (2004) Coarse woody debris in undisturbed and logged forests in the eastern Brazilian Amazon. Global Change Biology 10: 784-795.

Lewis SL, Brando PM, Phillips OL, van der Heijden GMF \& Nepstad D (2011) The 2010 Amazon drought. Science 331: 554.

Lopes BR \& Miola BTD (2010) Sequestro de carbono em diferentes fitofisionomias do Cerrado. SynThesis Revista Digital FAPAM 2: 127-143.
Lorenzi H(2002) Árvores brasileiras: manual de identificação e cultivo de plantas arbóreas do Brasil. $2^{\text {a }}$ ed. Instituto Plantarum de Estudos da Flora, Nova Odessa. 368p.

Luccas FS (2011) Estoques de necromassa em um cerrado sensu stricto e uma Floresta Ombrófila Densa Montana, no estado de São Paulo. Dissertação de Mestrado. Instituto de Botânica da Secretaria de Estado do Meio Ambiente, São Paulo. 100p.

Malhi Y \& Grace J (2000) Tropical forests and atmospheric carbon dioxide. Trends Ecology Evolution 15: 332337.

Malhi Y, Baker TR, Phillips OL, Almeida S, Alvarez E, Arroyo L, Chave J, Czimczik CI, Di Fiore A, Higuchi N, Killeen TJ, Laurance SG, Laurance WF, Lewis SL, Montoya LMM, Monteagudo A, Neill DA, Vargas PN, Patino S, Pitman NCA, Quesada CA, Salomão R, Silva JNM, Lezama AT, Martinez RV, Terborgh J, Vinceti B \& Lloyd J (2004) The above-ground coarse wood productivity of 104 Neotropical forest plots. Global Change Biology 10: 563-591.

Marimon BS, Felfili JM, Lima ES, Duarte WMG \& Marimon-Junior BH (2010) Environmental determinants for natural regeneration of gallery forest at the Cerrado/Amazonia boundaries in Brazil. Acta Amazonica 40: 107-118.

Marimon BS, Marimon-Junior BH, Feldpausch T, Oliveirasantos C, Mews HA, Lopez-gonzales G, Lloyd J, Franczak DD, Oliveira EA, Maracahipes L, Miguel A, Lenza E \& Phillips O (2014) Disequilibrium and hyperdynamic tree turnover at the forest-cerrado transition zone in southern Amazonia. Plant Ecology \& Diversity 7: 281-292.

Marimon-Junior BH \& Haridasan M (2005) Comparação da vegetação arbórea e características edáficas de um cerradão e um cerrado sensu stricto em áreas adjacentes em solos distróficos no leste de Mato Grosso, Brasil. Acta Botanica Brasilica 19: 913-926.

Medeiros MB (2002) Efeitos do fogo nos padrões de rebrotamento em plantas lenhosas, em campo sujo. Tese de Doutorado. Universidade de Brasília, Brasília. $121 \mathrm{p}$.

Medeiros MB \& Miranda HS (2005) Mortalidade pós-fogo em espécies lenhosas de campo sujo submetido a três queimadas prescritas anuais. Acta Botanica Brasilica 19: 493-500.

Mews HA, Marimon BS, Pinto JRR \& Silvério DV (2011a) Dinâmica estrutural da comunidade lenhosa em Floresta Estacional Semidecidual na transição Cerrado-Floresta Amazônica, Mato Grosso, Brasil. Acta Botanica Brasilica 25: 845-857.

Mews HA, Marimon BS, Maracahipes L, Franczak DD \& Marimon-Junior BH (2011b) Dynamics of the woody community of a typical cerrado in Northeastearn Mato Grosso. Biota Neotropica 11: 73-82.

Mota MC \& Torezan JMD (2013) Necromassa em reflorestamentos com espécies nativas da Mata Atlântica com 4, 6 e 8 anos de implantação. Hoehnea 40: 499-505. 
Nogueira EM, Fearnside PM, Nelson BW, Barbosa RI \& Keizer EWH (2008) Estimates of forest biomass in the Brazilian Amazon: new allometric equations and adjustments to biomass from wood-volume inventories. Forest Ecology and Management 256: 1853-1857.

Nordén B \& Paltto H (2001) Wood-decay fungi in hazel wood: species richness correlated to stand age and dead wood features. Biological Conservation 101: 1-8.

Oliveira B, Marimon-Junior BH, Mews HA, Valadão MBX \& Marimon BS (2016) Unraveling the ecosystem functions in the Amazonia-Cerrado transition: evidence of hyperdynamic nutrient cycling. Plant Ecology 217: 225-239.

Palace M, Keller M, Asner GP, Silva JNM \& Carlos $P$ (2007) Necromass in undisturbed and logged forests in the Brazilian Amazon. Forest Ecology and Management 238: 309-318.

Palace M, Keller M \& Silva H (2008) Necromass production: studies in undisturbed and logged Amazon Forests. Ecological Applications 18: 873884.

Palace M, Keller M, Hurtt G \& Frolking S (2012) A review of above ground necromass in tropical forests. In: Sudarshana P, Nageswara-Rao M \& Soneji JR (eds.) Tropical Forests, Online Publisher: 215-252.

Pan Y, Birdsey RA, Fang J, Houghton R, Kauppi PE, Kurz WA, Phillips OL, Shvidenko A, Lewis SL, Canadell JG, Ciais P, Jackson RB, Pacala SW, Mcguire AD, Piao S, Rautiainen A, Sitch S \& Hayes D (2011) A large and persistent carbon sink in the world's forests. Science 333: 988-993.

Ratter JA, Bridgewater S \& Ribeiro JF (2003) Analysis of the floristic composition of the Brazilian Cerrado vegetation III: comparison of the woody vegetation of 376 areas. Edinburgh Journal of Botany 60: 57-109.

Rebetez M, Renaud V, Von Arx G \& Dobbertin M (2012) Impact of forest cover on increases in temperature under the canopy. General Assembly Conference Abstracts 14: 4443.

Redin M, Miguel P, Denega GL, Lupatini M, Doneda A, Lorensi SE \& Franceschi SGD (2011) Impactos da queima sobre atributos químicos, físicos e biológicos do solo. Ciência Florestal 21: 381-392.

Reis SM, Lenza E, Marimon BS, Gomes L, Forsthofer M, Morandi PS, Marimon Junior BH, Feldpausch TR \& Elias F (2015) Post-fire dynamics of the woody vegetation of a savanna forest (Cerradão) in the Cerrado-Amazon transition zone. Acta Botanica Brasilica 29: 408-416.

Ribeiro JF \& Walter BMT (2008) As principais fitofisionomias do Bioma Cerrado. In: Sano SM, Almeida SP \& Ribeiro JF (eds.) Cerrado: Ecologia e Flora. EMBRAPA, Brasília. Pp. 151-212.

Roberto RL (2014) Densidade da madeira, biomassa aérea e estoque de carbono de árvores em três fitofisionomias da Transição Cerrado-Amazônia, Brasil. Dissertação de Mestrado. Universidade do Estado de Mato Grosso, Nova Xavantina, 43p.

Saiter FZ, Wendt T, Villela DM \& Nascimento MT (2009) Rain Forests: floristics. In: International commission on tropical biology and natural resources. Encyclopedia of life support systems (EOLSS). UNESCO, Oxford. Pp. 203-228.

Sayer EJ (2006) Using experimental manipulation to assess the roles of litter in the functioning of forest ecosystems. Biological Reviews 81: 1-31.

Stadtler EWC (2007) Estimativas de biomassa lenhosa, estoque e seqüestro de carbono acima do solo ao longo do gradiente de inundação em uma floresta de igapó alagada por água preta na Amazônia Central. Dissertação de Mestrado. Universidade Federal do Amazonas, Manaus. 57p.

Toledo JJ (2009) Influência do solo e topografia sobre a mortalidade de árvores e decomposição de madeira em uma floresta de terra-firme na Amazônia central. Tese de Doutorado. Instituto Nacional de Pesquisa da Amazônia, Manaus. 84p.

Valadão MBX, Marimon-Junior BH, Oliveira B, Lúcio NW, Souza MGR \& Marimon BS (2016) Biomass hyperdynamics as a key modulator of forest selfmaintenance in a dystrophic soil in the AmazoniaCerrado transition. Scientia Forestalis 44: 475-485.

Vale AT, Brasil MAM \& Leão AL (2002) Quantificação e caracterização energética da madeira e casca de espécies do cerrado. Ciência Florestal 12: 71-80.

Veloso HP, Rangel filho ALR \& Lima JCA (1991) Classificação da vegetação brasileira, adaptada a um sistema universal. Instituto Brasileiro de Geografia e Estatística, Rio de Janeiro. 124p.

Weedon JT, Cornwell WK, Cornelissen JHC, Zanne AE, Wirth C \& Coomes DA (2009) Global meta-analysis of wood decomposition rates: a role for trait variation among tree species? Ecology Letters 12: 45-56.

Zar JH (2010) Bioestatistical analysis. $5^{\text {th }}$ ed. Prentice Hall, New Jersey. 944p. 